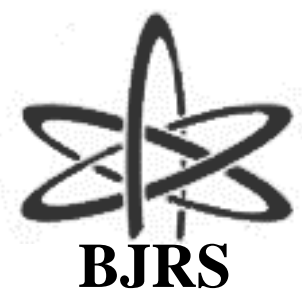

BRAZILIAN JOURNAL

$\mathrm{OF}$

RADIATION SCIENCES

08-01B (2020) 01-15

\title{
Desenvolvimento de ferramentas computacionais para otimizar a distribuição espacial de sementes na próstata em procedimentos de braquiterapia
}

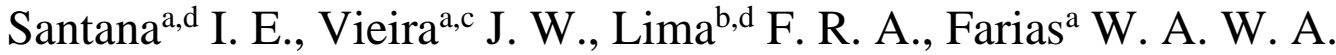 \\ ${ }^{a}$ Instituto Federal de Educação, Ciência e Tecnologia de Pernambuco, 50740-545, Av. Prof. Luís Freire, 500 - Cidade \\ Universitária, Recife - PE. \\ ${ }^{b}$ Centro Regional de Ciências Nucleares do Nordeste, 50730-120, Av. Prof. Luís Freire, 200 - Curado, Recife - PE. \\ ${ }^{c}$ Escola Politécnica de Pernambuco, 50720-001, R. Benfica, 455 - Madalena, Recife - PE. \\ ${ }^{d}$ Universidade Federal de Pernambuco, 50670-901, Av. Prof. Moraes Rego, 1235 - Cidade Universitária, Recife - PE.
}

e-mail do eufrazioivan@hotmail.com

\section{RESUMO}

A braquiterapia de próstata com baixa taxa de dose é um dos tratamentos que podem ser utilizados contra o câncer de próstata e consiste na aplicação de determinado número de fontes radioativas no interior da próstata. Contudo, as radiações emitidas por essas fontes, além de eliminar o tumor, podem causar danos em órgãos sadios considerados de risco (bexiga e reto). Logo, é necessário realizar um planejamento prévio e eficaz através de avaliações dosimétricas e isso pode ser feito através de Modelos Computacionais de Exposição (MCEs). A construção de MCEs, bem como a análise dos resultados gerados por eles, envolve a utilização de Ferramentas Computacionais (FCs). O objetivo desse trabalho foi desenvolver FCs para construir um MCE (MSUP_VOI_Prostata) e avaliar seus resultados. Esse MCE avalia o comportamento da dose de radiação, na próstata e nos órgaos de risco, quando diferentes blocos (VOIs), com diferentes distribuições aleatórias de fontes radioativas, são gerados em diferentes partes da próstata. Os protocolos de braquiterapia de próstata recomendam distribuir as sementes dentro da próstata de forma uniforme, na periferia ou de forma híbrida e irradiam a próstata como um todo. Foi visto que as FCs desenvolvidas nesse trabalho permitem gerar, através de 
técnica Monte Carlo, diversas distribuições aleatórias de fontes em toda a próstata ou em regiões específicas. Essas distribuições podem ser simuladas com o MSUP_VOI_Prostata e os resultados das simulações utilizados para selecionar a região da próstata e a distribuição de fontes que gere uma maior relação entre a dose na próstata e nos órgãos de risco.

Palavras-chave: Braquiterapia de próstata, modelos computacionais de exposição, ferramentas computacionais.

\footnotetext{
ABSTRACT

Low dose rate prostate brachytherapy is one of the treatments that can be used in prostate cancer and it consists in the application of certain number of radioactive sources inside the prostate. However, the radiation emitted from these sources can also cause damage in organs considered at risk (bladder and rectum). Therefore, it is necessary to perform a previous and good planning and do dosimetrics evaluations. This can be done by Exposure Computational Models (MCEs). The MCEs building and its results analysis is done by Computational Tools (FCs). The aim of this work was to implement FCs to build a MCE (MSUP_VOI_Prostata) an evaluate its results. This MCE evaluates the radiation dose in both the prostate and in the organs at risk when different blocks (VOIs), with different aleatory distribution of radioactive sources, are generated in different parts of the prostate. The protocols of prostate brachytherapy recommend three different types of sources distribution inside the prostate (uniform, peripherical and hybrid) and they irradiate all of the prostate. The FCs implemented in this work allows to generate, for Monte Carlo technique, various aleatory sources distribution in all of the prostate or in specific parts of it. These distributions can be simulated with MSUP_VOI_Prostata and its results used to select a prostate region and a source distribution that generate a high ratio between the dose in prostate and in the risk organs.
}

Keywords: Prostate Brachytherapy, Exposure computational models, computational tools. 


\section{INTRODUÇÃO}

O câncer de próstata é tido como um problema de saúde pública. No Brasil, de acordo com o Instituto Nacional do Câncer, ele é o segundo mais frequente em homens [1]. Um dos tratamentos que pode ser utilizado contra esse câncer é a braquiterapia de próstata com baixa taxa de dose. Esta, consiste na aplicação de determinado número de sementes radioativas no interior da próstata através de agulhas inseridas na região perineal. Contudo, as radiações emitidas por essas fontes podem causar danos em órgãos sadios considerados de risco (bexiga e reto) [2] e, para minimizar esses danos, deve-se realizar um planejamento prévio e eficaz através de avaliações dosimétricas.

Uma das formas de realizar avaliações dosimétricas é utilizar Modelos Computacionais de Exposição (MCEs). Estes são compostos, fundamentalmente, por um algoritmo simulador de determinada fonte radioativa, um fantoma computacional e um código Monte Carlo (MC) para realizar a interação da radiação com a matéria, bem como avaliar a energia depositada e grandezas de normalização em regiões de interesse.

A construção de MCEs bem como a análise dos resultados gerados por eles após as simulações, envolve a utilização de ferramentas computacionais (FCs). As FCs desenvolvidas pelo Grupo de Pesquisa em Dosimetria Numérica (GDN) têm sido concentradas, principalmente, nos softwares DIP (Digital Image Processing) [3] e MonteCarlo [4]. O DIP é um software que contém diversas FCs voltadas para construção de fantomas de voxels tais como: transformação de formato de imagens, compactação de imagens 2D para formação de imagens 3D, quantização, reamostragem, melhoramento e segmentação de imagens, entre outras. O MonteCarlo, por sua vez, contém FCs para construção, execução e análise de resultados de MCEs. Em 2017, Vieira [4] apresentou a última atualização desse software e, entre outras coisas, validou métodos MC de construção de imagens sintéticas binárias de ossos para substituir similares obtidos por microtomografia [5] de amostras reais. Estas imagens contêm informações sobre a distribuição das trabéculas ósseas em cinco regiões do esqueleto humano, necessárias para simular a interação de fótons e elétrons com os tecidos ósseos e a consequente deposição de energia.

Uma das etapas do planejamento em braquiterapia de próstata consiste em definir a distribuição geométrica das sementes radioativas dentro do órgão. Essa distribuição pode ser uniforme, 
periférica ou uniforme modificada [2] [6] [13]. Na distribuição uniforme, as sementes são colocadas em toda a próstata com distâncias fixas de $1 \mathrm{~cm}$ umas das outras em profundidade craniocaudal, alternando em planos pares e ímpares a cada $0,5 \mathrm{~cm}$ a colocação ântero-posterior e látero-lateral em 0,5 cm umas das outras. Na periférica, as sementes são colocadas somente na periferia da próstata com atividades mais altas. A uniforme modificada intercala planos com distribuições uniformes e periféricas. Cada uma das distribuições apresenta vantagens e desvantagens, e a uniforme modificada na maioria das situações clínicas satisfaz os requerimentos do radio-oncologista, do urologista e do físico [6]. Contudo, essas três distribuições utilizam todo o volume da próstata. Certamente a escolha de um volume de próstata específico para se inserir determinado número de sementes radioativas iria reduzir a probabilidade de danos nos órgãos de risco.

O objetivo desse trabalho foi implementar FCs para construir um MCE (MSUP_VOI_Prostata), analisar seus resultados e assim auxiliar na otimização de procedimentos de braquiterapia de próstata com baixa taxa de dose. Esse MCE permite realizar simulações utilizando várias distribuições aleatórias de sementes radioativas de I-125 localizadas em diferentes regiões da próstata, avaliar a dose de radiação na próstata e nos órgãos de risco para cada conjunto de semente simulado, e então selecionar um volume específico que gere uma maior razão entre a dose na próstata e nos órgãos de risco.

A maioria dos trabalhos de avaliação dosimétrica realizados em braquiterapia de próstata tem como foco as características das fontes utilizadas. Em 2013, Menezes [7] utilizou o fantoma masculino REX [8] e o código MC MCNP [9] para realizar um estudo dosimétrico em braquiterapia de próstata com sementes de I-125 e Pd-103. Em 2018, Justino e colaboradores [10] utilizaram um MCE composto pelo fantoma MASH3 e o código MC MCNP para realizar avaliações dosimétricas em braquiterapia de próstata e assim comparar as sementes radioativas de I 125 fabricadas pelo Instituto de Pesquisas Energéticas e Nucleares (IPEN) e o modelo 6711 Oncoseed da Amersham. Conforme esperado, a próstata recebeu a maior dose de radiação seguida da bexiga, cólon e gônadas.

Acredita-se que a seleção de uma região específica da próstata para inserir determinado número de fontes radioativas e uma configuração específica das fontes dentro da desse órgão pode tratar o tumor com mais eficácia e poupar ainda mais os órgãos de risco. 


\section{MATERIAIS E MÉTODOS}

\subsection{Materiais}

A realização deste trabalho esteve concentrada no Laboratório de Dosimetria Numérica (LDN) do Instituto Federal de Educação, Ciência e Tecnologia de Pernambuco (IFPE). Foi utilizado um notebook com sistema operacional Windows 10 Pro 64 bits, 16 GB de RAM e processador Intel(R) Xeon(R) E-2176M CPU @ $2.70 \mathrm{GHz}$ para implementar FCs a fim de desenvolver o MSUP_VOI_Prostata, realizar simulações dosimétricas e fazer a análise dos resultados. Neste notebook foram instalados os softwares RadiAnt; ImageJ; DIP; MonteCarlo; Visual Studio Community 2017; Geany; EGSnrc, Microsoft Office e as bibliotecas de classes BibliotecaJWV.dll e ArquivoDeDados.dll. Utilizou-se ainda o espectro discreto de energia do I-125 [11] e o MCE MSUP e fantoma MASH_SUP do Departamento de Energia Nuclear da Universidade Federal de Pernambuco (DEN/UFPE) [12].

\subsection{Métodos}

A construção do MSUP_VOI_Prostata se deu através de alterações realizadas no MCE MSUP [12]. Este é composto pelo fantoma MASH na posição supina (MASH_SUP) acoplado ao código Monte Carlo EGSnrc e contém 13 algoritimos de fontes externas e 1 para fonte interna (fonte 14). As alterações foram realizadas nos arquivos de input (.egsinp) e no código principal (.mortran) para determinar, entre outras coisas, o espectro utilizado, a quantidade e dimensão das sementes, o número do bloco de sementes utilizado e a forma como os resultados de saída deveriam ser salvos.

O MSUP_VOI_Prostata permite avaliar o comportamento da dose por atividade acumulada (D/Â), em mGy/MBq.s, na próstata e nos órgãos de risco quando se utilizam diferentes distribuições aleatórias de sementes (geradas através de técnica Monte Carlo) em diferentes partes de próstata ao longo dos três principais eixos anatômicos (X: laterolateral, Y: anteroposterior e Z: craniocaudal) e nos quatro respectivos sentidos (para direita, para esquerda, para frente, para trás, para cima, para baixo). O VOI corresponde a um paralelepípedo que pode conter parte ou todo o 
volume da próstata. A Figura 1 mostra o caminho e a imagem dos arquivos de entrada e de compilação contidos na pasta do MSUP_VOI_Prostata.

FCs foram implementadas, tanto no software MonteCarlo quanto no DIP, para gerar os seguintes arquivos de entrada para o MSUP_VOI_Prostata: um catálogo de espectros discretos de energia (EnergyDiscreteEspectra.txt), as coordenadas (x, y, z) dos voxels centrais das sementes radioativas dentro de cada VOI (SeedsBlocks__DirSen.txt) e os arquivos de input (.inp) para cada VOI a ser utilizado na simulação (MSUP_DirSen_IProstata.egsinp). Onde, Dir = direção (X, Y ou Z) e Sen $=$ sentido $(+$ ou -$)$. Os arquivos de input contêm todos os parâmetros necessários para iniciar a simulação, escritos em formato a ser lido no código MSUP_VOI_Prostata.mortran ${ }^{1}$.

A FC implementada para gerar o arquivo SeedsBlocks__DirSen.txt, se encontra no DIP em Estudos $\rightarrow$ Medicina $\rightarrow$ Braquiterapia $\rightarrow$ Criar arquivo SeedsBlocksI125.txt.... Ela lê o fantoma de entrada passado pelo usuário, solicita uma direção de partida $(\mathrm{X}+$ (direita), X- (esquerda), Y+ (frente), Y- (trás), Z+ (baixo), Z- (cima)) e requer os parâmetros descritos na Figura 3. O fantoma utilizado foi o MASH_SUP que contém voxels com $0,12 \mathrm{~cm}$ de aresta. Os parâmetros da Figura 3 foram colocados apenas para exemplificar a utilidade dessa FC e não representam um tratamento real.

Como a distribuição uniforme de sementes tende a gerar altas doses de radiação no centro da próstata e a periférica tende a subdosar essa mesma área [13], optou-se por utilizar uma distribuição aleatória de sementes e assim encontrar uma melhor configuração.

Seguindo os parâmetros da Figura 3, a FC precisa, de início, sortear a posição de 15 sementes, com 3 voxels cada, separadas entre si por no mínimo 2 voxels ( 5 x 5 x 7) e alinhadas na direção Z, dentro de um VOI que contém todo o volume da próstata. Para tanto, essa FC cria um fantoma SGI de saída com IDs $=0$ e com as mesmas dimensões do fantoma de entrada. Então, sorteia um voxel no VOI, candidato a voxel central de uma semente. Checa se ele possui o mesmo número identificador (ID) do órgão onde serão inseridas as sementes (39) e se seus vizinhos (5 x 5 x 7) são diferentes, para garantir que não haja sobreposição de sementes. Garantidas estas condições, o arquivo de saída recebe o ID voxel central (150) na localização sorteada. Os outros voxels da

\footnotetext{
${ }^{1}$ Do mesmo modo que a extensão .egsinp identifica um arquivo de entrada para o compilador do EGSnrc, a extensão .mortran identifica um código de usuário. Os arquivos .mortran devem ser escritos em passos (Figura 2) e contêm dois laços principais: o mais externo para ciclos de blocos informados no egsinp e o mais interno para ciclos de histórias da partícula [14]. Os retângulos em verde correspondem aos passos onde foram feitas alterações no código.
} 
semente (100) estarão em Z+1 e Z-1. Com essas informações a FC gera as localizações dos voxels centrais das sementes do VOI atual e escreve, adicionalmente, estes dados em uma variável interna de lista de texto. Serão adicionados nessa lista tantos blocos quanto forem possíveis observando a seguinte condição: com o passo e a direção fornecida, gerar o número de sementes fornecido dentro da próstata. Ao final da execução, a lista é salva em um arquivo de texto nomeado, por exemplo, SeedsBlocks_X+I125.txt, quando o usuário escolheu a direção horizontal no sentido das menores para as maiores posições de pixels (ver sistema de orientação do EGSnrc na Figura 4). Para o exemplo desse trabalho, foram gerados 24 VOIs em X+, 25 em X-, 25 em Y+, 23 em Y-, 20 em Z+ e 20 em Z-.

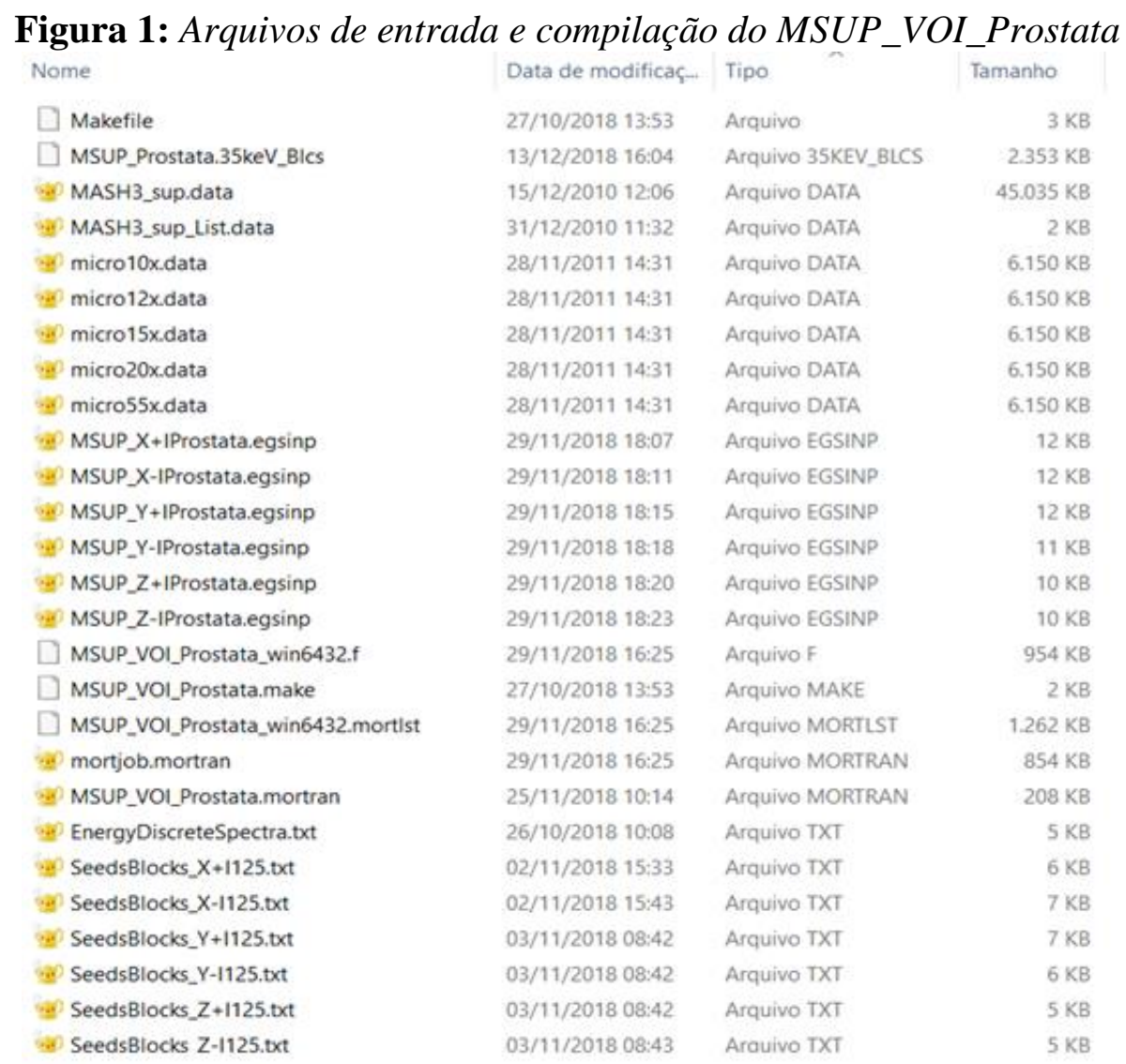

Para cada arquivo SeedsBlocks_DirSen.txt gerado foi criado um arquivo de entrada do tipo MSUP_DirSen_IProstata.egsinp. Os arquivos de entrada foram modificados para informar o número do VOI a ser lido dentro de cada arquivo SeedsBlocks_DirSen.txt, a quantidade e as 
dimensões das sementes no VOI que está sendo lido e o número do espectro discreto de energia a ser selecionado no arquivo EnergyDiscreteSpectra.txt durante a simulação. Como o objetivo do trabalho não foi avaliar as características da fonte utilizada e nem a distribuição de dose em diferentes regiões da próstata, os espectros foram salvos nesse catálogo sem levar em consideração a autoblindagem das fontes e a atenuação exercida pelo material que as envolve. Quando esses parâmetros forem avaliados, o espectro modificado será salvo dentro do catálogo para que possa ser utilizado na simulação.

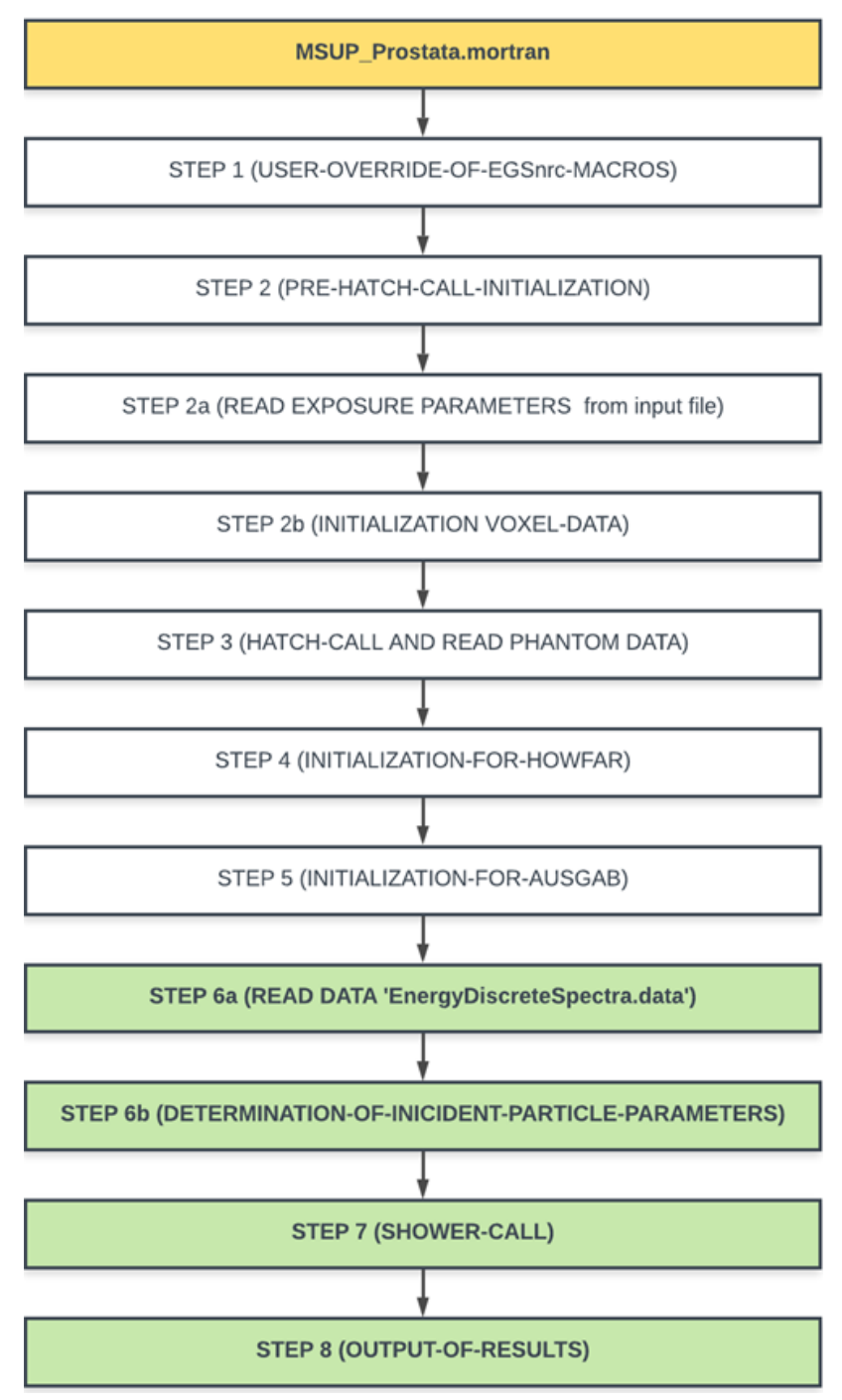

Figura 2: Esquema de passos do arquivo MSUP_Prostata.mortran 
Figura 3: Parâmetros para gerar blocos, com 15 sementes cada, ao longo de $X+$.

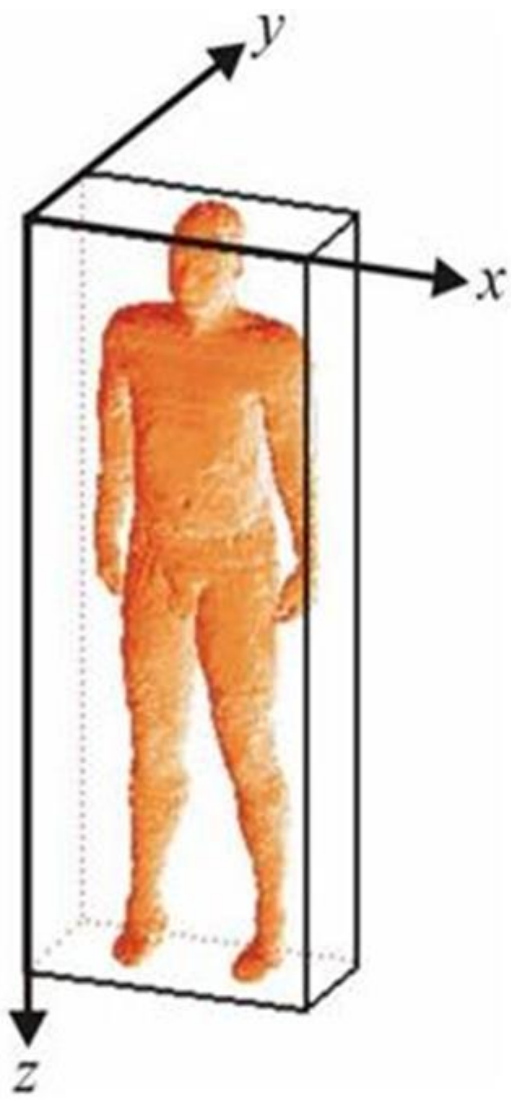

Figura 4: Fantoma MAX no sistema de coordenadas do EGS.

Fonte: VIEIRA, 2004.

No código de usuário MSUP_VOI_Prostata.mortran, de acordo com as informações contidas nos arquivos MSUP_X+IProstata.egsinp, o espectro selecionado é lido dentro do laço dos blocos no passo 6a de leitura de dados do esquema geral de códigos .mortan (Figura 2). Os dados (energias e suas probabilidades acumuladas) são transferidos para variáveis vetoriais internas, reais de 4 
bytes. No mesmo passo, os arquivos SeedsBlocks__DirSen.txt são lidos, e seus dados (coordenadas $\mathrm{x}, \mathrm{y}, \mathrm{z}$ dos voxels centrais de cada semente) transferidos para variáveis vetoriais internas, reais de 4 bytes.

No passo 6b (determinação dos parâmetros da partícula emergente), o código da fonte interna do MSUP [15] foi modificado para sortear de qual fonte de I-125 começa cada história e, dentro desta, de qual ponto o fóton emerge e qual a direção que ele toma. Além disso, um número aleatório (entre 0 e 1) é sorteado para definir a probabilidade acumulada atual e relacioná-la à energia inicial do fóton emergente. Este é o estado inicial de uma partícula na simulação.

No passo 7 (chamada da função shower), com estas 7 variáveis sorteadas (três coordenadas, três cossenos diretores e a energia em $\mathrm{MeV}$ ) a função shower é chamada para realizar os demais estados das partículas. Os resultados são salvos em variáveis matriciais 3D com as mesmas dimensões do fantoma, previamente definida para receber, cumulativamente, a energia depositada em cada voxel por história.

No passo 8 (saída de resultados), a matriz 3D é utilizada para transferir os dados para todos os arquivos de saída relacionados com dose (MSUP_NblcDirSenIProstata.35keV). Onde, Nblc $=\mathrm{N}^{\mathrm{o}}$ do bloco $(01,02 \ldots)$; Dir = direção $(\mathrm{X}, \mathrm{Y}$ ou Z); Sent = sentido $(+$ ou -).

Os resultados da simulação, obtidos com o MSUP_VOI_Prostata, consistem em vários arquivos do tipo MSUP_NblcDirSentIProstata.35keV.data, um para cada VOI de distribuição de sementes. Esses arquivos contém a relação de todos os órgãos segmentados no fantoma MASH_SUP e seus respectivos valores de dose por atividade acumulada (D/Â), em mGy/MBq.s. Eles foram unificados utilizando uma FC implementada no software MonteCarlo em Arquivos $\rightarrow$ Criar (organizar) arquivos de saída dos MCEs do GDN $\rightarrow$ Criar o ArquivosDados_DI.txt. O arquivo resultante foi denominado ArquivoDados_VOI_Prostata.txt. Ele foi compactado utilizando uma FC já implementada no software MonteCarlo e adicionado à pasta recursos do projeto ArquivosDeDados para que fossem lidos dentro do software MonteCarlo. Ainda no ambiente do MonteCarlo foi implementada uma nova FC em Análise gráfica e numérica $\rightarrow$ Dosimetria interna $\rightarrow$ Braquiterapia de próstata $\rightarrow$ Variação da posição de VOIs contendo sementes de I 125 no MCE MSUP..., para ler esses arquivos e gerar gráficos e tabelas que relacionam a D/Ã nos órgãos de interesse, com a posição, em cm, do VOI de cada distribuição de sementes, em relação à origem do sistema de coordenadas do EGSnrc, ao longo dos três eixos anatômicos. 


\section{RESULTADOS E DISCUSSÃO}

Nesta seção serão apresentados os resultados obtidos através dos testes realizados com as ferramentas implementadas. Foram feitas simulações com todos os VOIs gerados e todo o processo levou um tempo computacional de aproximadamente 48 horas. Contudo, esse tempo pode ser reduzido através de programação em paralelo. A utilização da técnica Monte Carlo para gerar diversas distribuições aleatórias de fontes dentro da próstata é importante pois é possível ir além dos três tipos de distribuições comumente utilizadas [2] [6] [13].

A Figura 5 apresenta uma janela secundária da FC utilizada para ler e apresentar os resultados obtidos com o MSUP_VOI_Prostata contendo dados de entrada, botões de clique e de marca, tabelas e gráficos de saída. É possível visualizar, em um mesmo gráfico, o comportamento da D/Ã em órgãos específicos e assim selecionar um VOI de interesse que gere uma maior relação entre a dose na próstata e nos órgãos de risco. Uma vez escolhido o VOI ideal, o usuário pode encontrar a posição de cada semente na prósta dentro do arquivo SeedsBlocks_DirSen.txt.

Apenas para exemplificar o funcionamento da FC implementada para analisar os resultados da simulação, optou-se por apresentar os valores de D/Ã obtidos nas simulações com o MSUP_VOI_Prostata ao longo de Z+. O gráfico da Figura 6 mostra a variação da D/Ã na próstata (PR), parede da bexiga (PB), testículos (TE) e parede do cólon (PC), ao longo de Z+ (da cabeça para o pé do fantoma). Observa-se, como esperado, que a D/Ã se matém aproximadamente constante na próstata, diminui na parede da bexiga e parede do cólon, e aumenta nos testículos. Para esse caso, a melhor distribuição de sementes seria o VOI que está a 89,64 cm da cabeça do fantoma (origem do sistema de coordenadas do EGSnrc), pois a redução nos valores de D/Ã nas paredes do cólon e bexiga são mais significativas que o aumento apresentado pelos testículos (Tabela 1). Contudo, é preciso avaliar a localização do tumor dentro da próstata para que a dose de radiação nele não seja insuficiente já que o VOI localizado na posição supracitada engloba apenas a parte final da próstata (ápice).

É importante destacar que, em uma situação real, deve-se avaliar todos os 6 sentidos possíveis e assim determinar o VOI ideal com base nos valores de dose desejado para cada órgão. 


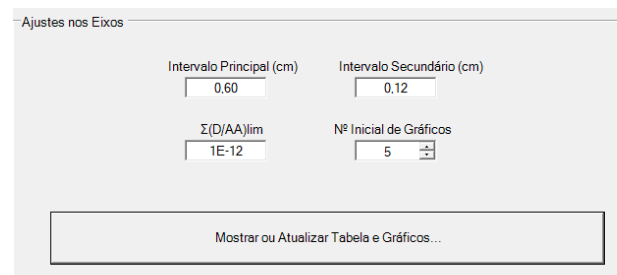

\section{D/AA (mGy/MBq.s) x Posiçăo do VOI (cm)}

- $Z$ (cm)/ÓR GLÅNDUL PAREDED PAREDED SEIOS. GR RINS FIGADO PULMÓES MU

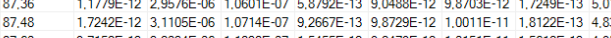

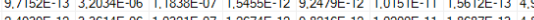

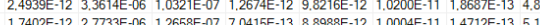

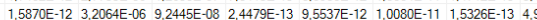

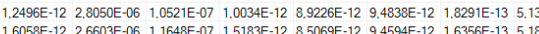

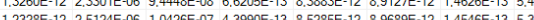

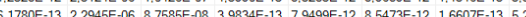

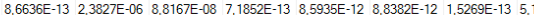

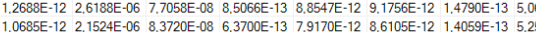
1.0685E-12 2.1524E-06 8.3720E- 08 6.J700E-13 7.9170E-12 8.6105E-12 $1.4055 E-13$ 5.2

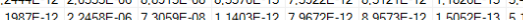

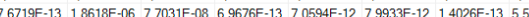

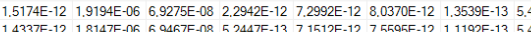

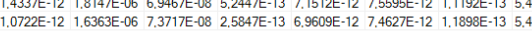

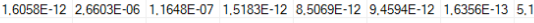

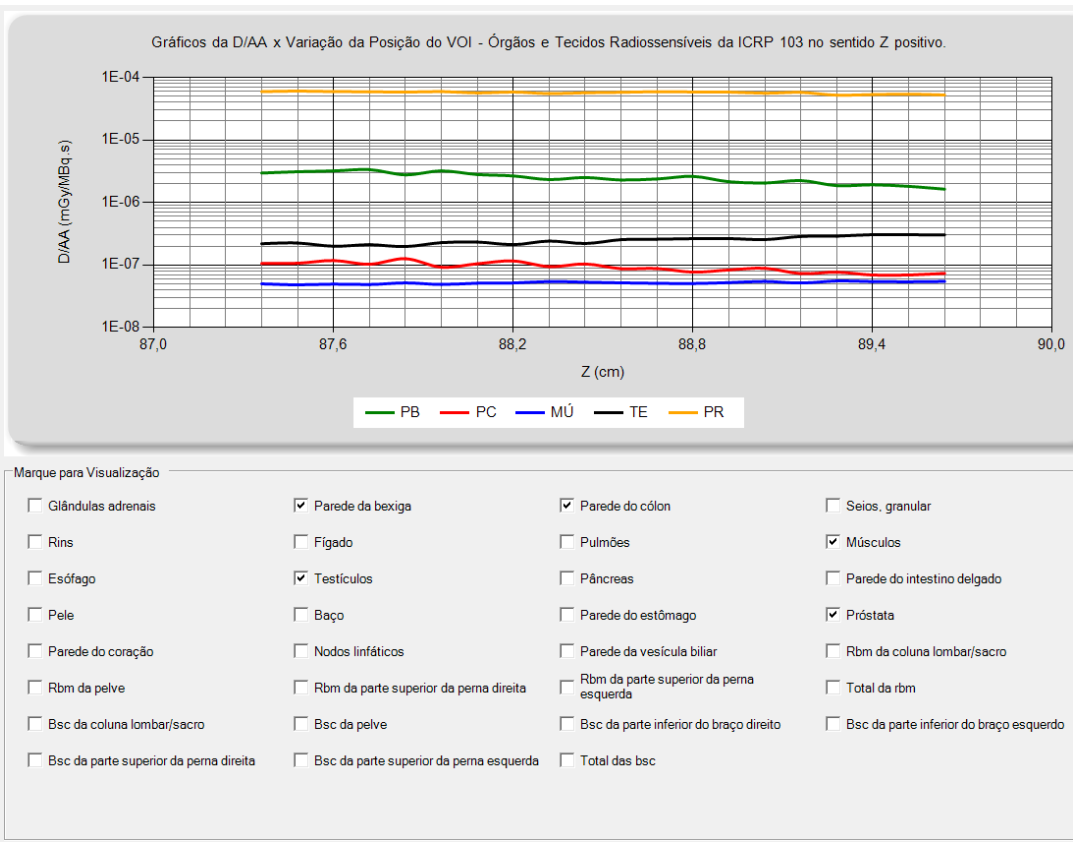

Figura 5: Ferramenta computacional para análise dos resultados do MSUP_VOI_Prostata

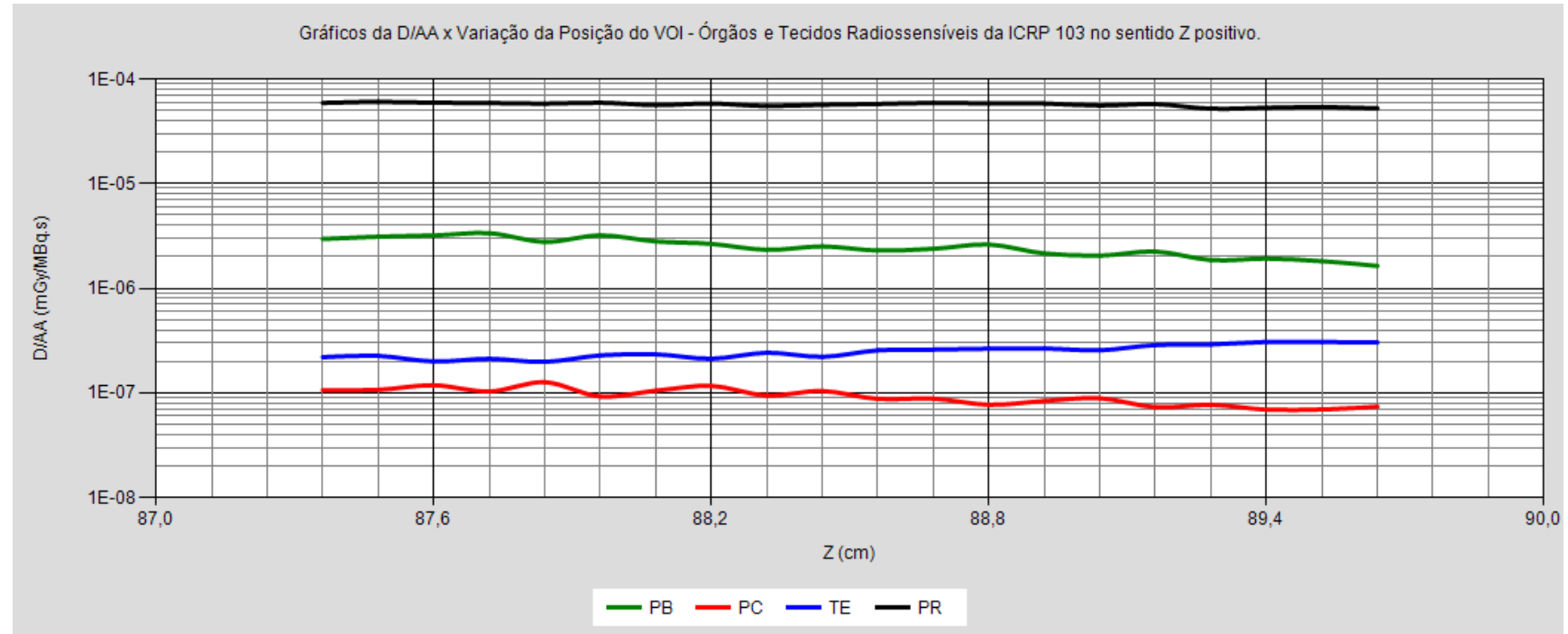

Figura 6: Valores de D/Ã na próstata, bexiga, cólon e testículos ao longo de Z+ 
Tabela 1: Valores de D/Ã para o primeiro e último VOI

\begin{tabular}{ccc}
\hline & \multicolumn{2}{c}{ Posição do VOI } \\
\cline { 2 - 3 } Órgão/tecido & $\mathbf{8 7 , 3 6} \mathbf{~ c m}$ & $\mathbf{8 9 , 6 4} \mathbf{~ c m}$ \\
\hline Próstata & $5,9197 \mathrm{E}-05 \mathrm{mGy} / \mathrm{MBq} . \mathrm{s}$ & $5,2379 \mathrm{E}-05 \mathrm{mGy} / \mathrm{MBq} . \mathrm{s}$ \\
Parede da bexiga & $2,9576 \mathrm{E}-06$ mGy/MBq.s & $1,6363 \mathrm{E}-06 \mathrm{mGy} / \mathrm{MBq} . \mathrm{s}$ \\
Testículos & $2,2001 \mathrm{E}-07$ mGy/MBq.s & $3,0402 \mathrm{E}-07$ mGy/MBq.s \\
Parede do cólon & $1,0601 \mathrm{E}-07$ mGy/MBq.s & $7,3717 \mathrm{E}-08$ mGy/MBq.s \\
\hline
\end{tabular}

\section{CONCLUSÕES}

Esse trabalhou mostrou que as FCs desenvolvidas nesse trabalho podem ser utilizadas para otimizar procedimentos de braquiterapia de próstata com baixa taxa de dose. Elas permitem gerar inúmeras distribuições de fontes radioativas em diferentes regiões da próstata e, através do MCE MSUP_VOI_Prostata, obter valores de D/Ã na próstata e em órgãos de risco para cada distribuição gerada. A partir de gráficos e tabelas é possível escolher uma região específica da próstata para inserir determinado número de sementes radioativas que gere uma maior razão entre a dose na próstata e nos órgãos de risco.

Para utilizar essas FCs com eficácia na prática hospitalar, é necessário realizar adaptações, principalmente na distribuição inicial de sementes e suas atividades. Também é necessário avaliar a localização do tumor na próstata para que o mesmo não receba uma dose de radiação insuficiente. Contudo, isso não implicaria em grandes esforços computacionais.

\section{AGRADECIMENTOS}

Os autores agradecem ao IFPE Recife, DEN/UFPE e CRCN/NE por disponibilizar seus ambientes e materiais para realização desse trabalho. 
Um agradecimento especial a Fundação de Amparo à Ciência e Tecnologia do Estado de Pernambuco (FACEPE) por disponibilizar recursos financeiros, através do projeto APQ 003630915, para compra de computadores utilizados nesse trabalho.

\section{REFERÊNCIAS}

[1] INCA - Instituto Nacional de Câncer José Alencar Gomes da Silva. Coordenação de Prevenção e Vigilância. Estimativa 2016: Incidência de Câncer no Brasil. Rio de Janeiro: INCA, 2015.

[2] PRENTICE, M.; NEI, W.; LEWIS, A.; DAVDA, R.; PAYNE, H. Brachytherapy in prostate cancer: techniques and clinical outcomes. Trends in Urology \& Men's Health. 2018.

[3] VIEIRA, J. W.; LIMA, F. R. A. A Software to Digital Image Processing to be Used in the Voxel Phantom Development. Cellular and Molecular Biology, vol. 55, 2009, 16-22 p.

[4] VIEIRA, J. W. MonteCarlo - Um Software para Uso em Avaliações Dosimétricas das Radiações Ionizantes. Recife: IFPE, 2017, 130 p.

[5] KRAMER, R.; CASSOLA, V. F.; VIEIRA, J. W.; KHOURY, H. J.; OLIVEIRA LIRA, C. A.; ROBSON BROWN, K. Skeletal Dosimetry Based on $\mu$ CT Images of Trabecular Bone: Update and Comparisons. Physics in Medicine and Biology, vol. 57, No. 12, 2012.

[6] SALVAJOLI, J. V.; SOUHAMI, L.; FARIA, S. L. Radioterapia em Oncologia. São Paulo: Atheneu, 2013, 1312 p. 88.

[7] MENEZES, A. F., Estudo Dosimétrico de Braquiterapia de Próstata com Sementes de I125 e Pd-103 Usando Modelagem Computacional. Tese de doutorado. Rio de Janeiro: UFRJ, 2013, $130 \mathrm{p}$.

[8] ICRP. International Commission on Radiological Protection. Realistic reference phantoms: an ICRP/ICRU joint effort. A report of adult reference computational phantoms. ICRP Publication 110. Ann. ICRP 39, 2009.

[9] PELOWITZ, D. B. MCNPX User's Manual, Version 2.7.0, Report LA-CP-11- 00438. Los Alamos National Laboratory; 2011. 
[10] JUSTinO, P. C.; SANTOS, W. S.; SANTOS, C. J.; NEVES, L. P.; BELINATO W.; PERINI, A. P. Avaliação Computacional das Doses em Braquiterapia para tratamento de câncer de Próstata com uma Nova Semente de 125I. XI Simpósio de Engenharia Biomédica, Uberlândia, 2018.

[11] CHU, S. Y. F.; EKSTROM, L. P.; FIRESTONE, R. B. The Lund/LBNL Nuclear Data Search. Version 2.0, February 1999.

[12] DEN/UFPE - Departamento de Energia Nuclear da Universidade Federal de Pernambuco, Disponível em: <http://caldose.org/> Acesso em 02 Set. 2019.

[13] NATH, R.; BICE, W. S.; BUTLER, W. M.; CHEN, Z.; MEIGOONI, A. S.; NARAYANA, V. ; RIVARD, M. J.; YU, Y. AAPM Recommendations on Dose Prescription and Reporting Methods for Permanent Interstitial Brachytherapy for Prostate Cancer. Report of AAPM Task Group 137, December 2009.

[14] KAWRAKOW, I.; MAINEGRA-HING, E.; ROGERS, D. W. O.; TESSIER, F.; WALTERS, B. R. B., The EGSnrc Code System: Monte Carlo Simulation of Electron and Photon Transport. NRCC Report PIRS-701. National Research Council of Canada, Ottawa, Canadá, 2017.

[15] VIEIRA, J. W. Construção de um Modelo Computacional de exposição para cálculos dosimétricos utilizando o código Monte Carlo EGS4 e fantomas de voxels. Tese de doutorado. Recife: UFPE, 2004, 101 p. 